\title{
PADRONIZAÇÃO DE SUFIXOS PARA POLITIPOS
}

\author{
ERNEST H. NICKEL* \\ Tradução do original em inglês "STANDARDIZATION OF POLYTYPE SUFFIXES" realizada com permissão da IMA por
}

\section{DANIEL ATENCIO**}

A Comissão de Novos Minerais e Nomes de Minerais (CNMNM) da Associação Mineralógica Internacional tem publicado recomendações sobre vários assuntos ligados à nomenclatura mineralógica, inclusive nomenclatura de politipos (e.g. Nickel \& Mandarino 1990). Em essência, politipos são distinguidos por símbolos alfanuméricos, adicionados ao nome raiz e ligados a ele por hífen. A parte numérica do símbolo representa a periodicidade das camadas e a parte alfabética, em itálico, representa o sistema cristalino. Por exemplo, wurtzita-4// é um politipo hexagonal da wurtzita com periodicidade de 4 vezes a dimensão $c$ da wurtzita original. Anteriormente à publicação destas diretrizes, vários outros símbolos para politipos foram utilizados na literatura mineralógica e, em um esforço para padronizar a nomenclatura de politipos, os membros da CNMNM decidiram que os nomes usados previamente deverão ser modificados de acordo com a prática corrente, que, por sua vez, segue as sugestões da União Internacional de Cristalografia (Guinier et ai. 1984). Os nomes para politipos na literatura que requerem modificações são os relacionados a seguir:

\author{
Nome original Nome atual \\ anandita-20r anandita-20 \\ gageíta-17c gageíta-1A \\ hilgardita-17c hilgardita-1A \\ hilgardita-37c hilgardita-1A \\ pirofilita-17c pirofilita-1A \\ safirina-1 $T c$ \\ Tc safirina-1A Merlino(1973) \\ Referência \\ Filutetal. (1958) \\ Ferraris et al (1987) \\ Ghose (1985) \\ Ghose(1985) \\ Wardle \& Brindley \\ (1972) \\ tyretskita-17c tyretskita-1A Ghose (1985) \\ wollastonita-ir wollastonita-1A Henmi et al. (1978) \\ wollastonita-3r wollastonita-3A Henmi et al. (1983) \\ wollastonita-47 wollastonita-4A Henmi et al. (1983) \\ wollastonita-5r wollastonita-5A Henmi et al. (1983) \\ wollastonita-?r wollastonita-7A Henmi et al. (1978)
}

É muito provável que a literatura mineralógica contenha exemplos adicionais de símbolos para politipos que devam ser revisados e adequados às presentes recomendações. Os símbolos a serem utilizados são os seguintes: cúbico, $\mathrm{C}$; hexagonal, $H$; romboédrico, /?; trigonal, $T$; tetragonal, $Q$ (quadrático); ortorrômbico, $O$; monoclínico, $M$; e triclínico, $A$ (anórtico).

\section{Referências}

Ferraris, G., Mellini, M., Merlino, S. (1987) Electron-diffraction and electron-microscopy study of balangeroite and gageite: Crystal structures, polytypism, and fibre texture. American Mineralogist 72, 382-391.

Filut, M.A., Rule, A.C. and Bailey, S.W. (1985) Crystal structure refinement of anandite-20r, a barium-and sulfur-bearing trioctahedral mica. American Mineratogist 70, 1298-1308.

Ghose, S. (1985). A new nomenclatura for the borate minerais in the hilgardite $(\mathrm{Ca} 2 \mathrm{BsO} 9 \mathrm{Cl} . \mathrm{H} 2 \mathrm{O})$ - tyretskite $\left(\mathrm{CaaBsOgOH} .^{\wedge} \mathrm{O}\right)$ group. American Mineralogist 70, 636-637.

Guinier, A., Bokij, G.B., Boll-Dornberger, K., Cowley, J.M., Durovic, S., Jagodzinski, H., Krishna, P., De Wolff, P.M., Zvyagin, B.B., Cox, D.E., Goodman, P., Hahn, Th., Kuchitsu, K., Abrahams, S.C. (1984) Nomenclature of polytype structures. Reportof the International Union of Crystallography Ad-Hoc Committee on the Nomenclature of Disordered, Modulated and Polytype Structures. Acta Crystallographica, A40, 399-404

Henmi, C., Kusachi, L, Kawahera, A., Henmi, K. (1978) 77wollastonite from Fuka, Okayama Prefecture. Mineralogical Journal 9, 169-181.
Henmi, C., Kawahara, A., Henmi, K., Kusachi, I., Tekeuchi, Y. (1983) The 37, 4T and 5T polytypes of wollastonite from Kushiro, Hiroshima Prefecture, Japan. American Mineralogist 68, 156-163.

Merlino, S. (1973) Polymorphism in sapphirine. Contributions to Mineralogy and Petrology 41, 23-29.

Nickel, E.H. \& Mandarino, J.A. (1990) Procedimentos envolvendo a Comissão de Novos Minerais e Nomes de Minerais da IMA, e diretrizes sobre nomenclatura mineral. Revista Brasileira de Geociências 20, 302-317 (Também publicado em outros periódicos).

Wardle, R. \& Brindley, G.W. (1972) The crystal structures of pyrophyllite, $\mid T c$, and of its dehydroxylate. American Mineralogist 57,732-750.

Manuscrito NB -17

Recebido em 30 de junho de 1998

Revisão dos autores em 20 de julho de 1998 Revisão aceita em 30 de julho de 1998 